\title{
Topical Ectoine Application in Children and Adults to Treat Inflammatory Diseases Associated with an Impaired Skin Barrier: A Systematic Review
}

\author{
Marion Kauth $\cdot$ Olga V. Trusova
}

Received: October 26, 2021 / Published online: January 17, 2022

(C) The Author(s) 2022

\begin{abstract}
Introduction: Inflammatory skin diseases are a significant burden on affected patients. Inflammation is caused by various stress factors to the epidermis resulting in, e.g., dryness, redness, and pruritus. Emollients are used in basic therapy to restore the natural skin barrier and relieve symptoms. A systematic review was performed to evaluate the efficacy and safety of ectoine-containing topical formulations in inflammatory skin diseases characterized by an impaired skin barrier.

Methods: A systematic review was carried out in PubMed, the Cochrane Library, and Microsoft Academic up to October 2021. Inclusion criteria were ectoine-containing topical formulations (creams, emollients) used for (adjuvant) therapy of inflammatory skin diseases. Clinical studies of any design published in any language were included.
\end{abstract}

Results: A total of 230 references were screened for eligibility, of which six were selected for

M. Kauth $(\bowtie)$

Department of Research and Development, Bitop

AG, Carlo-Schmid-Allee 5, 44263 Dortmund,

Germany

e-mail: Marion.Kauth@bitop.de

O. V. Trusova

Department of Clinical Therapy with the Course on

Allergy and Immunology, Pavlov University, Saint

Petersburg, Russia inclusion in the review (five studies on atopic dermatitis and one study on prevention and management of retinoid dermatitis). The application of topical formulations containing $5.5-7.0 \%$ ectoine positively influenced skin dryness and, consequently, pruritus and dermatitis-specific scores in patients with atopic dermatitis. Especially in infants and children, who belong to the most frequently affected group, the formulations were well-tolerated when applied for up to 4 weeks. In studies where ectoine was used as an adjuvant therapy, application was associated with a decreased need for pharmacological therapy and also improved the effectiveness of, e.g., topical corticosteroids. In patients undergoing isotretinoin therapy, ectoine was as effective as dexpanthenol in reducing retinoid dermatitis or improving symptoms.

Conclusion: Ectoine is an effective natural substance with an excellent tolerability and safety profile, representing a beneficial alternative as basic therapy or to increase the efficacy of the pharmacological treatment regimen for patients with inflammatory skin diseases, including infants and children.

Keywords: Atopic dermatitis; Children; Ectoine; Eczema; Extremolyte; Hydration; Pediatric; Retinoid dermatitis; Skin barrier; TEWL 


\section{Key Summary Points}

The objective of this systematic literature review was to summarize available performance and safety data on topically applied ectoine-containing formulations for the treatment of inflammatory skin diseases in children and adults characterized by an impaired skin barrier.

Six clinical studies were identified through the systematic literature search, including atopic dermatitis and retinoid dermatitis as indications.

Topical formulations with up to $7 \%$ ectoine were safe and efficient in pediatric and adult populations when used in longterm application for up to 6 months.

Data suggest that ectoine-containing formulations enhance topical pharmacological therapy in inflammatory skin diseases and, thus, reduced the frequency and/or amount of pharmacological therapy.

\section{INTRODUCTION}

The body is protected by a stratified epithelial cellular sheet called epidermis [1]. The epidermis is a complex barrier protecting the human body from external invasion and water loss via a physical and chemical barrier [1, 2]. Inflammatory skin diseases such as atopic dermatitis or psoriasis are considered major public health issues with a globally increasing prevalence, especially in industrialized regions $[2,3]$. The inflammation is attributed to a disorder of the epidermal barrier function of the skin [1].

A frequent inflammatory skin disease is atopic dermatitis, also known as atopic eczema or neurodermatitis $[2,4]$. The global prevalence is estimated to be $15-20 \%$ of children and $1-3 \%$ of adults $[5,6]$. This medical condition classically manifests as dry skin and relapsing eczema accompanied by pruritus, often leading to sleep disturbances. Pruritus is the main symptom and is an essential characteristic on which diagnosis of atopic dermatitis is based [2]. Itching of the affected area further irritates the skin and worsens existent pruritus [2]. Disease severity is determined by, e.g., the classical composite score "SCORing of Atopic Dermatitis" (SCORAD), which evaluates affected skin areas and intensity (erythema, edema, oozing, excoriation, lichenification, dryness) and subjective symptoms including pruritus and sleep disturbance [4]. Symptoms usually start during early infancy or childhood and can worsen within the first years of life due to accompanying food allergies, asthma, and/or allergic rhinitis. This is referred to as "atopic march" $[1,2]$. The onset of atopic dermatitis commonly occurs before 5 years of age, whereas the highest onset is reported between the ages of 3 and 6 months. Approximately $60 \%$ of patients develop symptoms within the first year of life, and 90\% within 5 years of life [6]. Quality of life is significantly impaired in patients affected by atopic dermatitis, caused not only by symptoms (pruritus and sleep disturbance) but also by social, academic, and occupational factors [7]. Regarding disease pathology, it is hypothesized that surrounding triggers lead to a weakened skin barrier and consequently negatively impact the effective protection of the skin. It has also been suggested that an impaired skin barrier precedes the clinical condition that leads to immune dysregulation [2,8]. The basic therapies are educational programs, emollients, bath oils, and avoidance of clinically relevant allergens, but topical corticosteroids or calcineurin inhibitors can be included in the treatment regimen for mild to moderate cases. Severe cases might need hospitalization, biologic therapy, and/or systemic immunosuppression [4, 9]. Basic therapy with regular emollients and appropriate skin care combined with antiinflammatory treatment, while avoiding contact with triggering allergens and irritating agents, aim to improve clinical symptoms and avoid symptom aggravation [4]. Consequently, dry skin is managed by epidermis care by using moisturizing preparations to rehydrate the skin and further improve inflammation [10]. 
Other forms of inflammatory skin diseases can occur because of contact to irritants (e.g., retinoid dermatitis) or as an autoimmune disease (e.g., psoriasis). Retinoid dermatitis is a common side effect in topical tretinoin therapy of severe acne. Patients experience erythema, scaling, dryness, burning, and pruritus after implementing tretinoin treatment [11]. In vivo studies in mice demonstrated that retinoid treatment induces desquamation by degrading corneodesmosomes. Corneodesmosomes are important adhesion structures in epidermal cohesion [11], and are essential for intact epidermal barrier function [12]. One cornerstone of the management of various forms of inflammatory skin diseases includes application of humectant substances that alleviate symptoms by improving skin hydration [13]. Since its discovery in 1985 [14], the hydrating and inflammation-reducing properties of ectoine have been subject to intensive research.

Ectoine, (S)-2-methyl-1,4,5,6-tetrahydropyrimidine-4-carboxylic acid, is a cyclic amino acid naturally produced by extremophile microorganisms living under conditions of extreme salinity, drought, irradiation, $\mathrm{pH}$, and temperature $[14,15]$. The amino acid protects cells against chemical and physical noxa [14]. Furthermore, ectoine has been well assessed within the last few years, and has been shown to protect cell membranes of affected cells from dryness-induced reactions and subsequent inflammatory reactions by forming a water shell (ectoine hydrocomplex) around proteins [16]. The effect of increased hydration of the cell membrane also improves mobility and function of the lipid layer, leading to a better resistance against extreme conditions [17] and a reduction of transepidermal water loss (TEWL) [16]. As a result, these factors improve the impaired barrier function of the skin, protecting the skin against penetration of chemical and physical noxa [16-18]. Interestingly, key factors in symptom aggravation of atopic dermatitis include external factors such as irritants and allergens; therefore, strengthening the barrier function is a key strategy in overcoming symptoms of atopic dermatitis.

Within the last few years, the beneficial properties of ectoine have been demonstrated in several nonclinical [16-31] and clinical studies involving different indications [32-41] in which ectoine-based formulations were successfully used to alleviate symptoms of skin impairments or mucous membranes at different sites of the human body, such as nose and eyes, mouth or airways. Moreover, topical administration of ectoine has been reviewed for various indications, such as allergic rhinoconjunctivitis [42] and upper airway inflammation [43], as well as irritation and inflammations of the eye surface [44]. However, there is no summary of clinical evidence of ectoine-containing formulations on inflammatory skin diseases characterized by an impaired skin barrier. Therefore, a systematic literature review was performed to evaluate currently available clinical data on the management of inflammatory skin diseases concerning topical ectoine application.

\section{METHODS}

\section{Systematic Literature Review}

The systematic literature review adhered to the Preferred Reporting Items for Systematic Reviews and Meta-Analyses (PRISMA) guidelines $[45,46]$. The literature search was conducted in MEDLINE (via PubMed), the Cochrane Collection Central Register of Clinical Trials (CENTRAL), and Microsoft Academic to identify clinical trials on topical ectoine application as cream or emollient for the management of inflammatory skin diseases (search date: 1 October 2021). The search strategy included a combination of controlled vocabulary terms $(\mathrm{MeSH})$ and free text search terms for the substance and disease of interest. The following search/MeSH terms were queried in the respective databases:

- PubMed: ("ectoine"[All Fields] OR “ectoin"[All Fields] OR "ectoines"[All Fields] OR "dermaveel" [All Fields] OR "perfectoin" [All Fields]) AND ("skin"[MeSH Terms] OR "skin"[All Fields] OR "dermal"[All Fields] OR "dermally"[All Fields] OR "topical" OR "topically"[All Fields] OR "topicals"[All Fields]). 
- CENTRAL: [All text] ectoin OR [All text] Dermaveel OR [All text] Perfectoin (word variations).

- Microsoft Academic: "ectoine skin cream" [Filter: Ectoine [Top topics]]; "dermatitis dry skin ectoin" [Filter: Dermatology [Top topics]; Dry skin [Top topics]; Atopic dermatitis [Top topics]; Medicine [Top topics]); "perfectoin"; "dermaveel".

In addition, the bibliographies of included references identified in the search were screened. No restrictions were applied for the year of publication or type of study. References in any language were included in the analysis and, if necessary, publications were translated to English for further evaluation.

\section{Identification and Selection of Studies}

The review was conducted using a prespecified protocol. Predefined eligibility criteria were the use of the Population (nonrestricted), Interventions (ectoine-containing formulations), Comparisons (other interventions), and Outcomes (PICO) [47]. Studies with interventions but without comparator intervention or when ectoine-containing formulations were used as adjuvant therapy were also eligible for inclusion in this review. Publications not related to dermal application of ectoine or to the defined pathologies by the study were excluded from the analysis.

\section{Data Extraction}

Relevant information extracted from eligible studies were study design and methods, patient characteristics, intervention details (e.g., dosing, schedule, follow-up duration, ectoine concentration of the formulation, additional treatment regimens), and efficacy and safety outcomes, as well as time points for outcome assessments.

\section{Compliance with Ethics Guidelines}

This systematic review is based on previously conducted studies and does not contain any new studies with human participants or animals performed by any of the authors.

\section{RESULTS}

\section{Study Selection}

The search strategy identified 211 records after removing duplicates. Seven publications were screened for full-text eligibility after title/abstract screening. One reference was excluded as it was the trial registration (ClinicalTrials.gov) of the study published by Marini et al. [35]. In total, six references were eligible for inclusion in the systematic review (Fig. 1). Detailed information on the study design and methods, patient characteristics, intervention details (e.g., dosing, schedule, follow-up duration, ectoine concentration of the formulation, additional treatment regimens), and efficacy and safety outcomes is presented in Table 1 .

\section{Study Design and Study Population}

Six studies were assessed, of which three were designed as prospective, open label studies [48-50], one as a prospective, open label study with control pairs [51], one as a randomized, nonblinded study [52], and one as randomized, intraindividual double-blinded study [35]. Certainty of evidence was graded according to the Grading of Recommendations Assessment, Development and Evaluation (GRADE) framework and ranged from very low (one study) to moderate certainty (three studies) (Table 1). The studies predominately included patients with atopic dermatitis [35, 48-51], while one study evaluated the prevention and management of retinoid dermatitis after pharmacological acne treatment [52]. The study duration (including follow-up) varied from 2 to 4 weeks for atopic dermatitis and 6 months for retinoid dermatitis. Sample sizes ranged from 30 [49] to 242 [48], resulting in 525 recruited patients. Studies were performed on various continents and in several countries including Asia (Hong Kong) [49] and Europe (Germany, Poland, Russia) [35, 48, 50-52]. 


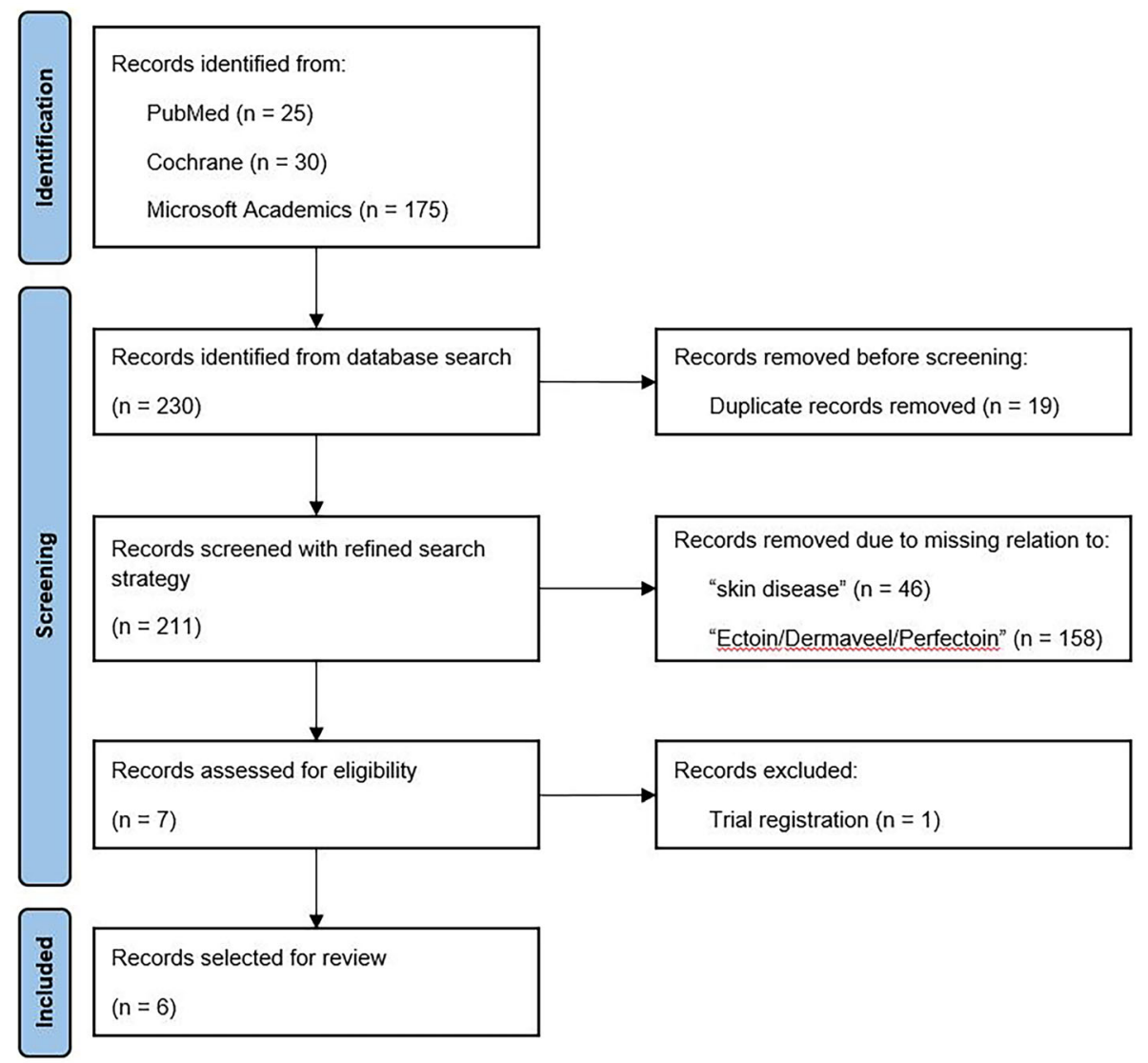

Fig. 1 PRISMA flow diagram of the systematic literature review

\section{Treatment Characteristics}

The applied formulations contained between $5.5 \%$ [48] and 7\% ectoine [35, 49-52]. Creams or emollients were used in all studies including the brand products Dermaveel, Perfectoin, and EHK02-01 creams (all by bitop AG, Witten, Germany). All formulations are based on basic creme formulations including other components also being defined as emollients or moisturizer. A full list of ingredients is provided in the legend of Table 1. Evaluated administration regimens included daily or twice daily application of the ectoine-containing formulation. The application in four studies was combined with other treatment regimens [48-51] including histamine antagonists [48, 49], topical corticosteroids [48-51], and calcineurin inhibitor $[48,51]$. For the prevention and management of retinoid dermatitis, the ectoine-containing formulation was combined with standard isotretinoin therapy [52].

\section{Efficacy of Ectoine-Containing Topical Formulations}

\section{Atopic Dermatitis}

Most assessed patients in the evaluated clinical studies suffered from atopic dermatitis (447 of 525 patients). The average age of patients ranged from 1.9 years [50] to 33.3 years old [35], presenting a mix of pediatric and adult populations. Three studies solely focused on the pediatric population [49-51]. One study only reported the mean age for the two included cohorts [50]. Of the 525 included patients, 455 were treated with an ectoine cream.

Most studies $(N=4)$ defined the disease severity of atopic dermatitis according to SCORAD $[48,51]$ or objective SCORAD index 


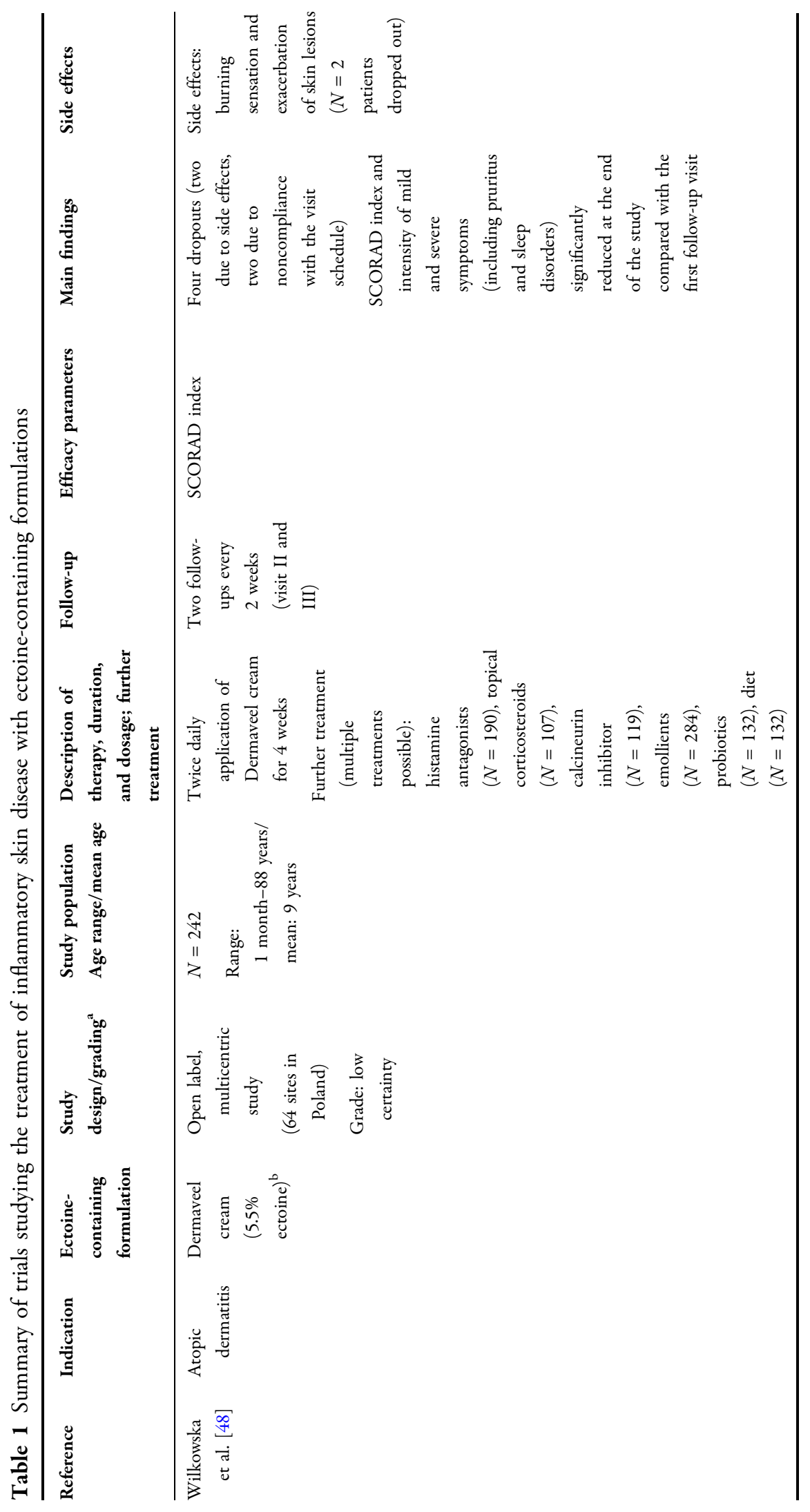




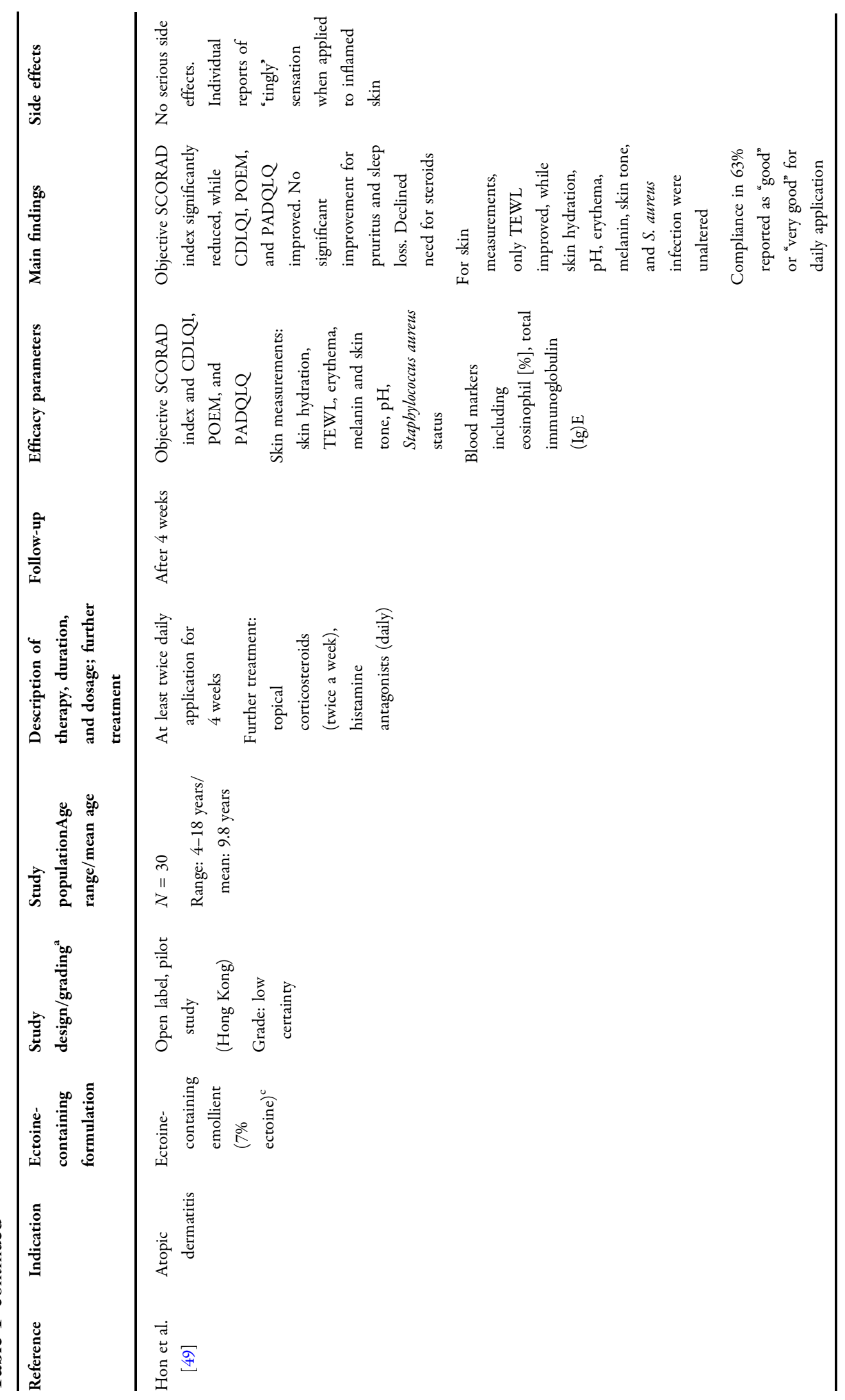




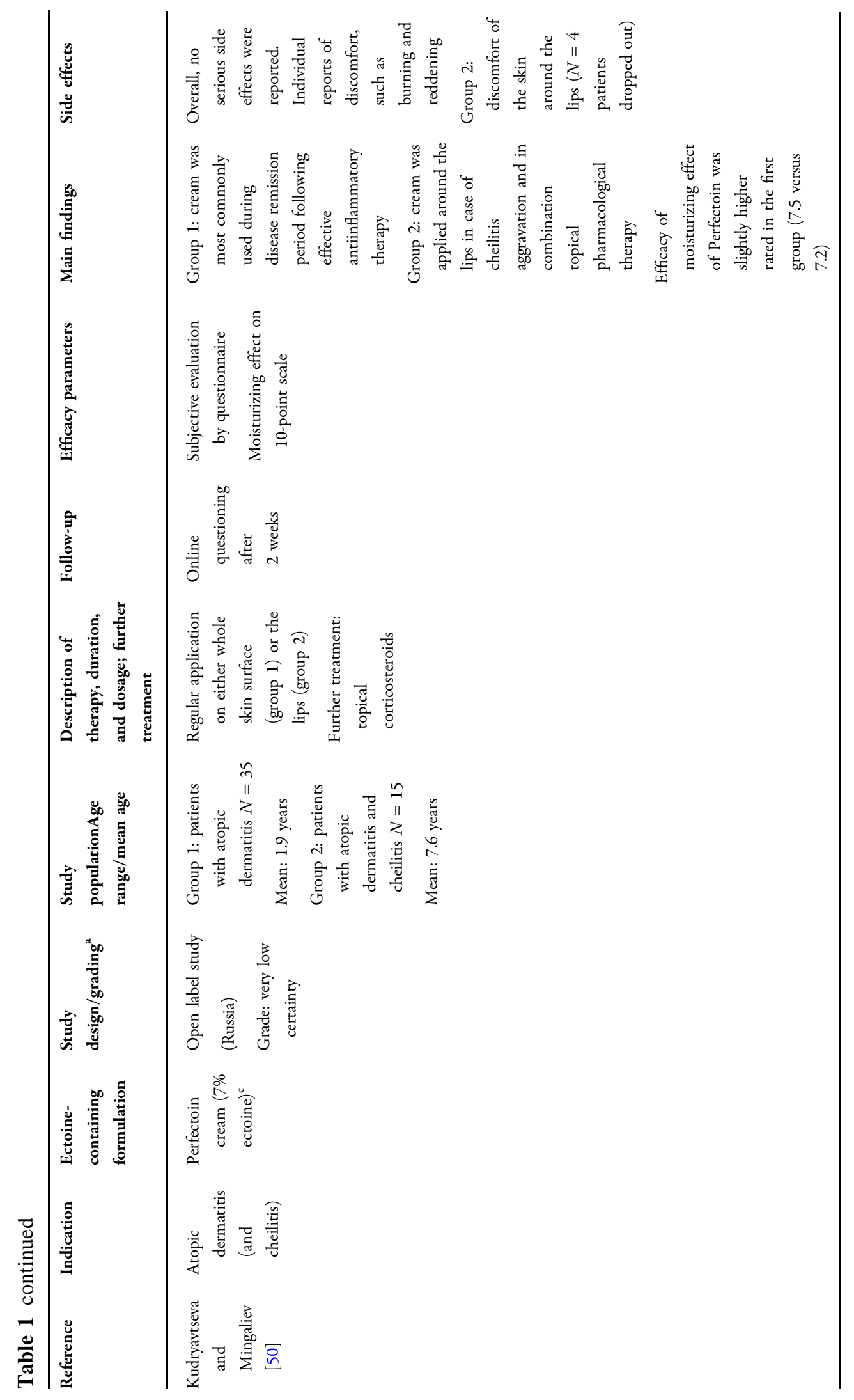




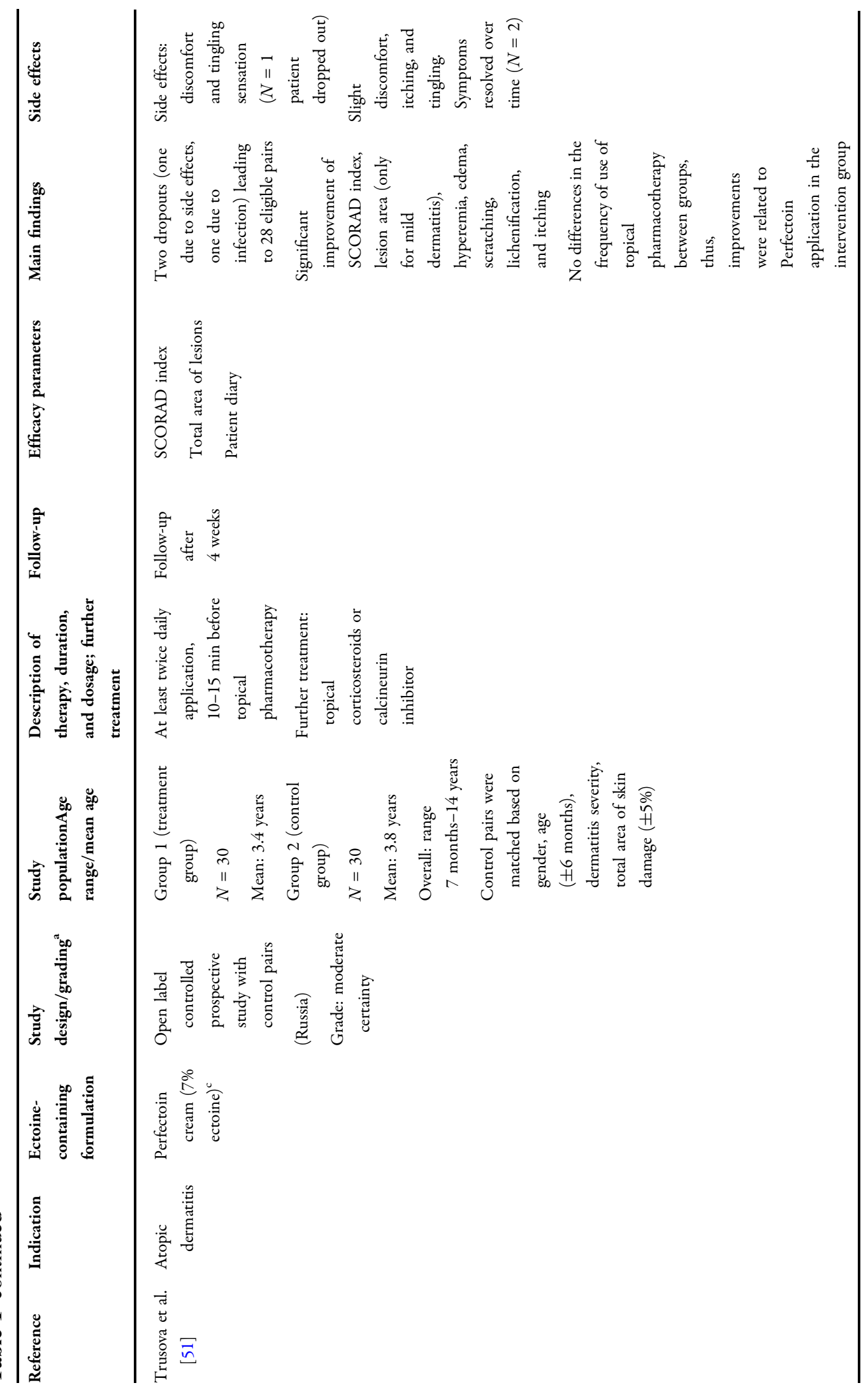




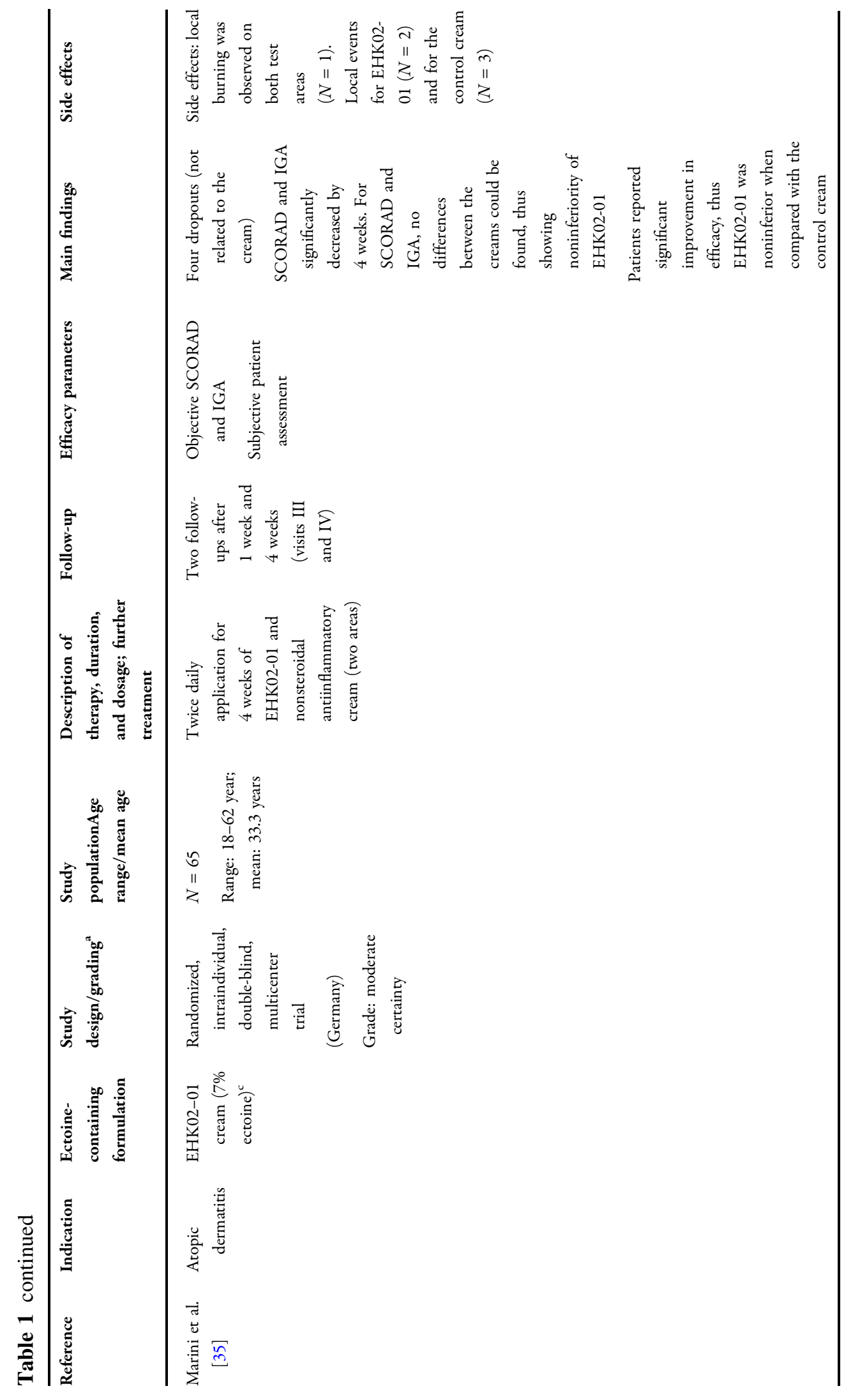




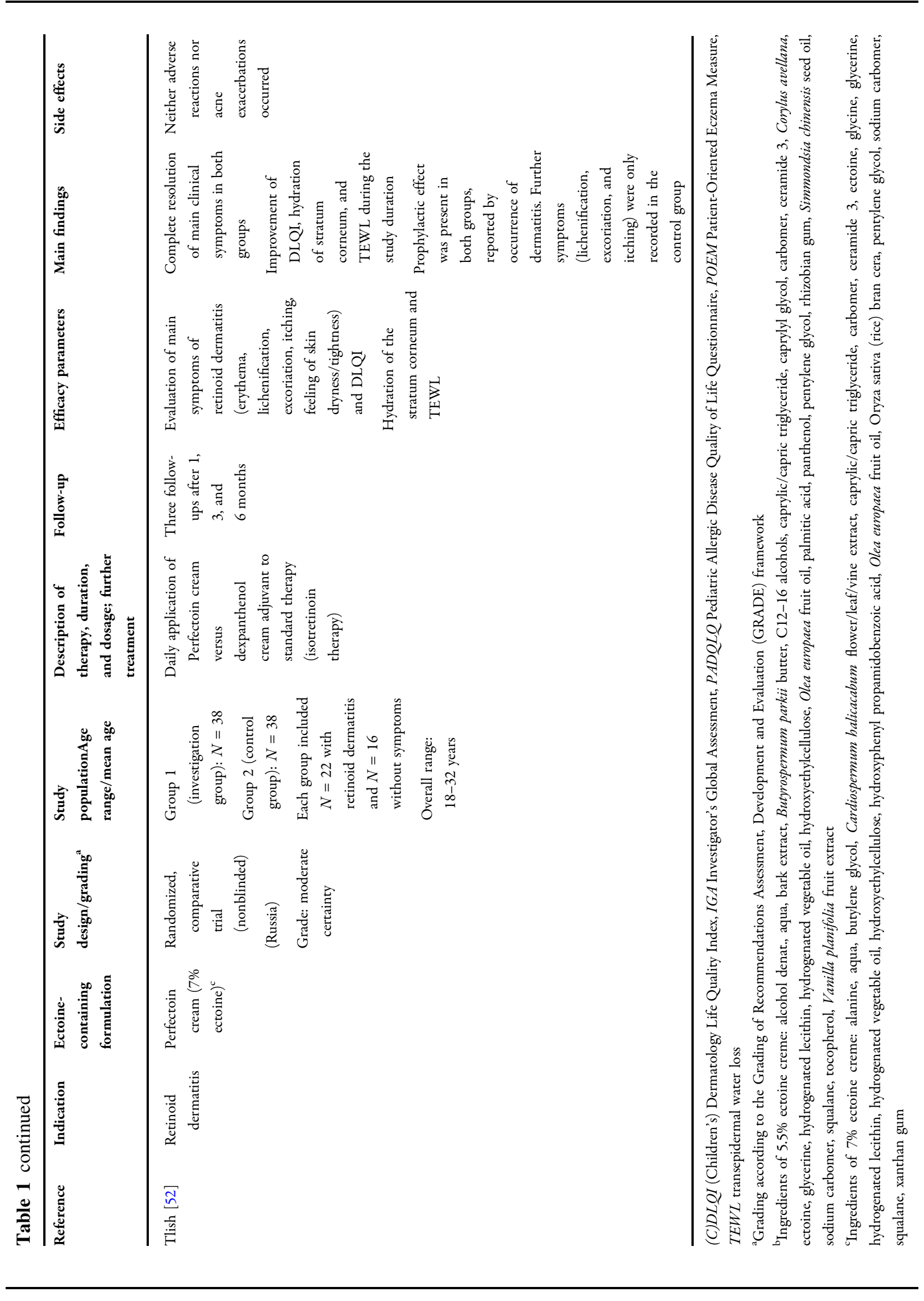


$[35,49]$. Only one study provided subjective evaluation by questionnaires [50].

Dermaveel, a 5.5\% ectoine cream, was assessed in a multicentric study with 242 patients recruited at 64 sites in Poland [48]. The age range of patients was from 1 month to 88 years, while the mean age was 9 years, suggesting that the majority was a pediatric study population. The cream was applied twice daily for 4 weeks and combined with several other treatment regimens (Table 1). A total of 238 patients completed the study period of 4 weeks and were included in the analysis. The SCORAD index reduced from 42 to 25 after 2 weeks and to 15 after 4 weeks $(p<0.05$ between individual visits). Further, the pruritus intensity significantly decreased from 5.6 to 2.2 $(p<0.05)$ and rating of sleep disorders reduced from 3.9 to $1.7(p<0.05)$. The authors additionally reported that the number of patients with severe or mild intensity of all symptoms was lower after 4 weeks when compared with the previous follow-up visit after 2 weeks, indicating a continuous improvement of dermatitis symptoms. Consequently, the number of patients with regression of lesions markedly increased at follow-up visits. Dry skin in moderate to severe lesions reduced from 53\% to $12 \%$ and from $39 \%$ to $2 \%$, respectively. The performance of the cream formulation was assessed as "very good" and "good" by $83 \%$ of patients, as "fair" by $14 \%$, and as "unsatisfactory" by $3 \%$ [48].

Trusova and colleagues performed a similar study in St. Petersburg, Russia, but evaluated a higher concentration ectoine cream (Perfectoin, $7 \%$ ectoine) in addition to the standard topical pharmacotherapy, in a solely pediatric population (7 months-14 years) [51]. The study design also included a control group only receiving standard topical pharmacotherapy; subjects were matched based on gender, age ( \pm 6 months), dermatitis severity, and total area of skin damage $( \pm 5 \%)$ for higher evidence. The mean age in both groups was 3.4 years versus 3.8 years. SCORAD index and total area of lesions were compared with the control group after 4 weeks of application of Perfectoin to persistent skin lesions of mild to moderate atopic dermatitis. Twenty-eight eligible pairs were available at follow-up for assessment. In patients with mild dermatitis (13 pairs), SCORAD index significantly improved from 16.6 to 10.7 in the intervention group and from 18.6 to 17.9 in the control group $(p=0.02)$. Simultaneously, lesion areas decreased from $5.5 \%$ to $2.0 \%$ in the intervention group and from $6.0 \%$ to $4.9 \%$ in the control group $(p=0.001)$. Moreover, significant improvements were reported for hyperemia (by 67\%; $p=0.001$ ), edema (by 31\%; $p=0.030$ ), scratching (by 43\%; $p=0.040$ ), lichenification (by $100 \% ; p=0.001$ ), and itching (by $71 \%$; $p=0.010)$. In patients with moderate dermatitis (15 pairs), SCORAD index improved from 26.7 to 15.0 in the treatment group when compared with the control group (25.6-19.4; $p=0.015)$. Similar to the pairs with mild dermatitis, lesion area (by $41.3 \% ; p=0.020$ ), hyperemia (by $44.0 \% ; p=0.022$ ), edema (by $28.0 \% ; p=0.03$ ), scratching (by $56.0 \% ; p=0.03$ ), lichenification (by $81.7 \% ; p=0.03$ ), and itching (by $67.4 \%$; $p=0.01$ ) significantly improved during the 4 week study period. In all groups, an increase or only little improvement of dryness outside of lesions was reported. This was expected because the study period was in the midst of the fall-winter period and skin dryness deteriorates in these months. No significant differences in the frequency of use of topical pharmacotherapy agents during the course of the study was described when compared with the control pairs. Thus, it was considered that the improvements observed in the intervention group were largely associated with the addition of the $7 \%$ ectoine cream in the therapy regimen [51].

An assessment of an identical study duration (4 weeks) and treatment regimen (application twice daily) was performed by Hon and coauthors in Hong Kong, Asia [49]. Ectoine (7\%) was applied in addition to topical corticosteroids or histamine antagonists. Thirty pediatric patients were recruited, with a mean age of 9.8 years, similar to the study population in the study by Wilkowska and colleagues [48]. Objective SCORAD index significantly reduced from 30.6 to 25.0 after 4 weeks $(p=0.002)$. Especially, the Children's Dermatology Life Quality Index (CDLQI) greatly improved from 9.9 to 9.2 
( $p=0.097$, statistically not significant), the Patient-Oriented Eczema Measure (POEM) from 15.4 to $11.6(p=0.035)$, and the Pediatric Allergic Disease Quality of Life Questionnaire (PADQLQ) from 39.0 to $30.6(p=0.017)$. In addition, data suggested a reduced need for corticosteroids $[3.8$ versus 3.1 days/week $(p>0.05)]$ and antihistamines $[1.7$ versus 0.8 days/week $(p=0.0059)]$. No significant improvements were reported for pruritus and sleep loss. For skin measurements, TEWL improved ( $p=0.035$ ) during the study duration, while skin hydration, $\mathrm{pH}$, erythema, melanin, skin tone (individual typology angle), and status of Staphylococcus aureus infection were reported to be unaltered. Compliance for daily application was rated as "good" or "very good" in $63 \%$ of patients. The authors compared the obtained results with previously generated data of similar emollients (Restoradom, Ezerra, and Ezerra Plus). The patient population was younger, but efficacy and acceptability among emollients were similar [49].

Marini and colleagues also evaluated the twice daily application of $7 \%$ ectoine cream for 4 weeks in 65 patients, including intraindividual controls (application of nonsteroidal antiinflammatory cream, Atopiclair) [35]. Patients' age ranged from 18 to 62 years, with a mean age of 33.3 years. Hence, this was the only study where children were not included. Objective SCORAD and Investigator's Global Assessment (IGA) describing staging atopic dermatitis severity were assessed. SCORAD significantly decreased from 7.5 to 5.3 after 1 week and to 3.8 after 4 weeks in the investigation group (Wilcoxon test baseline versus week 1 and baseline versus week 4 , respectively; each $p<0.0001$ ). Further, IGA scores were significantly reduced by $0.6-2.2$ points after 1 week and to 1.6 points after 4 weeks (Wilcoxon test baseline versus week 1 and baseline versus week 4, respectively; each $p<0.0001)$. Statistical testing for noninferiority revealed no differences between the creams for the primary endpoint SCORAD, thus demonstrating noninferiority of the ectoinecontaining formulation.

Patients rated pruritus decrease (assessed on an analogue visual scale from $0=$ no pruritus to $100 \mathrm{~mm}=$ worst pruritus) as significant, with
$24.19 \mathrm{~mm}$ after 1 week and $22.8 \mathrm{~mm}$ after 4 weeks (initially $46.8 \mathrm{~mm}$ ) (Wilcoxon test baseline versus week 1 and baseline versus week 4 , respectively; $p<0.0001)$. Atopiclair is approved by the United States Food and Drug Administration (FDA) for relief of itch, burning, and pain associated with mild to moderate atopic dermatitis [35]. In summary, this randomized, comparator-controlled, intraindividual, double-blind, multicenter trial provided evidence that the topical application of $7 \%$ ectoine cream to lesional skin of patients with mild to moderate atopic dermatitis markedly reduced the clinical severity of atopic dermatitis, regardless of whether it was measured by SCORAD, IGA, or self-assessment.

Kudryavtseva and Mingaliev extended their research question to other skin conditions associated with atopic dermatitis, i.e., cheilitis [50]. Cheilitis is a medical condition characterized by inflammation of the lips, often associated with aggravation of dermatitis symptoms. The first pediatric patient cohort included 35 children suffering from atopic dermatitis, with a mean age of 1.9 years. The second cohort consisted of 15 children affected by atopic dermatitis and cheilitis, with a mean age of 7.6 years. Both cohorts applied 7\% ectoine cream for 2 weeks before filling out an online questionnaire regarding the moisturizing effect of the cream. The cream was applied on either the whole skin surface (cohort 1) or the lips (cohort 2). The questionnaire revealed that the cream was most commonly used during disease remission following effective antiinflammatory therapy in the first cohort. The moisturizing efficacy of $7 \%$ ectoine cream was rated as strong, with 7.5 points (on a 10-score system with $0=$ no effect to $10=$ good effect) in this cohort. In the second cohort, the cream was applied around the lips in case of cheilitis aggravation and in combination with topical corticosteroid therapy. In this cohort, efficacy was rated similarly high, with 7.2 points [50].

\section{Retinoid Dermatitis}

Ectoine use for retinoid dermatitis as a secondary skin condition during isotretinoin therapy of moderate and severe acne was evaluated either for management of symptoms 
during the pharmacological therapy $(N=44 ; 22$ patients per group) or for prevention of symptoms $(N=32 ; 16$ patients per group). In this study, 7\% ectoine cream (intervention group) was compared with dexpanthenol cream (control group) [52]. The age range of patients in the prevention and management of retinoid dermatitis study was 18-32 years. Retinoid dermatitis severity was assessed by evaluating the main symptoms including erythema, lichenification, excoriation, itching, feeling of skin dryness, and Dermatology Life Quality Index (DLQI) [52]. All clinical symptoms and DLQI resolved after 6 months of treatment in both groups. Hydration of stratum corneum and TEWL increased simultaneously, with regression of clinical symptoms within the first months; especially, excoriation and itching completely resolved. Skin hydration index strongly increased by $15.1 \%(184.9 \mu \mathrm{S}$ versus $161.3 \mu \mathrm{S} ; p<0.05)$ in the investigation group during the first month, compared with an increase of $7.3 \%$ in the control group $(172.4 \mu \mathrm{S}$ versus $160.1 \mu S ; p<0.05)$. Statistical analysis of the difference between the visit time points is not provided in the study publication. TEWL decreased by $23.9 \%$ in the treatment group $\left(6.7 \mathrm{~g} / \mathrm{m}^{2} / \mathrm{h}\right.$ versus $\left.8.9 \mathrm{~g} / \mathrm{m}^{2} / \mathrm{h} ; p<0.05\right)$ and by $18.2 \%$ in the control group $\left(7.2 \mathrm{~g} / \mathrm{m}^{2} / \mathrm{h}\right.$ versus $\left.8.6 \mathrm{~g} / \mathrm{m}^{2} / \mathrm{h} ; p<0.05\right)$. During the subsequent months of therapy, no significant changes in these parameters were observed in either group, indicating the effectiveness of the prophylactic use of emollients during systemic isotretinoin therapy. After 6 months, DLQI reduced from 26 points in both groups, to 4 and 9 points in the treatment and control groups, respectively [52].

The prophylactic effect of the creams was evaluated by occurrence of dermatitis, which was reported to be $31.3 \%$ (5 of 16) in the intervention group and $53.5 \%$ (9 of 16) in the control group. As a result, a stronger prophylactic effect was demonstrated for the ectoine cream. Again, no statistical analysis was provided regarding the efficacy of either treatment. In addition, lichenification, excoriation, and itching were recorded in the control group but not in the treatment group [52].

\section{Safety}

Safety endpoints were included in all evaluated studies. Most side effects were discomfort such as burning sensation [35, 48, 50], tingly sensation $[49,51]$, reddening [50], and exacerbation of skin lesions [48]. In most cases, discomfort was only observed after the first application [50] and resolved over time [50, 51].These observations are consistent with the side effects mentioned in the instructions for use of the marketed ectoine-containing creams. In two adolescents and two patients aged 2 years with cheilitis secondary to dermatitis aggravation, use of $7 \%$ ectoine cream was discontinued because of discomfort of the skin around the lips after application [50]. Overall, seven out of 455 patients dropped out because of side effects related to the application of the ectoine-containing formulation $(N=2$ [48]; $N=4$ [50]; $N=1[51])$.

In the randomized, intraindividual doubleblinded trial comparing a nonsteroidal cream with ectoine-containing cream for the management of atopic dermatitis, side effects occurred equally in both treatment arms. In one patient, local burning was observed on both test areas. Two further events were reported for the investigated cream and three for the control cream [35], thus indicating no higher risk of side effects for $7 \%$ ectoine when compared with the control cream.

\section{DISCUSSION}

The occurrence of inflammatory skin diseases is associated with an increased production of carbon dioxide and exhaust gases, such as diesel exhaust particles, that result in air pollution and high-allergen environments, as often seen in urban areas of industrialized countries [1, 53]. Thus, it is not surprising that the prevalence of inflammatory skin diseases is constantly rising. Inflammatory skin diseases have a significant impact on the affected patients, not only medically but also socioeconomically [2]. Emollients and moisturizers are often recommended as first-line therapy to improve skin hydration and reduce symptoms such as itching or stinging. 
Mild antiinflammatory properties of the emollients can further reduce reliance on topical pharmacological therapy [13]. This systematic literature review was undertaken to evaluate the efficacy of ectoine-containing topical formulations for their hydrating and inflammation-reducing properties in the management of skin diseases with an impaired skin barrier. Furthermore, this review was performed to widen the availability of ectoine-referencing literature for the English-speaking community, since foreign language literature is limited to qualitative translation.

Interestingly, the study population included in this systematic review reflected a mix of pediatric and adult populations. Regarding atopic dermatitis, three out of five studies solely included infants and children [49-51], reflecting the high prevalence in this population, as reported in various studies and reviews $[5,6]$. Since acne and its treatment is a clinical condition in teenagers and adults, retinoid dermatitis is only found in adult patients undergoing acne treatment using systemic isotretinoin [52].

Considering the management of skin dryness and respective symptoms of atopic dermatitis, a SCORAD score or objective SCORAD score was evaluated in four out of five studies $[35,48,49,51]$. The score significantly decreased after application of the ectoine cream, implying an improved quality of life. This is consistent with reduced pruritus symptoms $[35,48,51]$, reduced need for itching, and further improvement of inflammatory symptoms such as hyperemia and edema [51]. Marini and colleagues compared the $7 \%$ ectoine formulation with the commercially available Atopiclair, which has antiinflammatory properties [35]. With regard to objective SCORAD and pruritus, noninferiority of the ectoinecontaining cream could be demonstrated in intraindividual controls [35]. In a Cochrane review on emollients and moisturizers for eczema treatment, it was concluded that Atopiclair significantly improved disease severity, decreased itching, and achieved more frequent satisfaction when compared with vehicle treatment [13]. Although no vehicle group was included in the study by Marini and colleagues, it can be assumed that the application of ectoine-containing cream is more effective than vehicle, based on the noninferiority of $7 \%$ ectoine formulation to Atopiclair. Overall, the positive influence of emollients or moisturizers in the management of dermatitis symptoms has been verified in various studies, as summarized by van Zuuren and colleagues [13]. Moisturizer use resulted in lower flares and prolonged time to flare [13]. Concerning retinoid dermatitis, promising results of $7 \%$ ectoine cream compared with standard therapy using dexpanthenol cream could be demonstrated [52].

Especially, results regarding the combination of ectoine-containing formulations with pharmacotherapies are of interest, since data from a meta-analysis suggest that emollients and moisturizers reduce the use of topical corticosteroids and increase the efficiency of topical active treatment [13]. In two studies evaluated here in pediatrics only, a reduced need for pharmacological therapy was reported, indicating that the amount of corticosteroids needed to achieve similar reductions in dermatitis severity could be reduced by applying ectoinecontaining formulations $[49,51]$.

Ectoine is a bacteria-derived extremolyte with the ability to protect proteins and biological membranes from damage caused by extreme environmental conditions such as heat, UV light, high osmolarity, or dryness $[14,15]$. The stabilization effect on the barrier function of the skin cells has led to the hypothesis that ectoine increases the resistance of impaired epidermis and improves its recovery by increased hydration and decreased TEWL [16]. Thus, the influence of ectoine on skin dryness is of particular interest in this review. Overall, the results from clinical trials presented in this review are consistent with the already published mode of action model of ectoine. However, skin hydration and the moisturizing effect of ectoine were only explicitly evaluated in three out of six studies, in which two studies objectively measured TEWL and/skin hydration $[49,52]$ and one study only subjectively rated the moisturizing effect [50]. Interestingly, although TEWL improved in the study described by Hon and colleagues, skin hydration did not alter within the 4-week study duration [49]. 
This was attributed to the seasonal drier skin in fall-winter [49]. Ectoine application (7\%) for 6 months led to improved hydration of the outer layer of the epidermis, the stratum corneum, and TEWL [52]. Similarly, dry skin improved by $51 \%$ in the study population described by Wilkowska and coauthors [48]. Additionally, a subjective evaluation supported the hydration effect of ectoine-containing formulations [50].

A key factor in symptom aggravation of, e.g., atopic dermatitis, includes external factors such as irritants and allergens. Hence, the reported clinical outcomes are in line with the results of nonclinical studies in rats and mice on the inflammation-reductive properties of ectoine, which allow insights into the underlying mechanisms of action. It was demonstrated that ectoine prevents lung inflammation induced by carbon nanoparticles via a mechanism involving the stabilization of macromolecules located at the outer cell surface rather than an interaction with the external particles [18]. Additionally, ectoine was shown to reduce allergic sensitization by preventing migration of antigen-loaded dendritic cells to the draining lymph nodes. This effect might be produced by either a direct action on dendritic cells or by suppression of neutrophilic inflammation [29].

Overall, there is evidence that topically administered ectoine improves subjective and clinical status of patients affected by inflammatory skin diseases. However, these findings should be viewed cautiously as they are based on a limited number of studies and patients in each indication, especially retinoid dermatitis. Patient population, application regimes, and treatment duration were also heterogenic between the studies. In addition, some of the studies might be underpowered because of their small patient numbers. This was also reflected in the low grading score of some studies. Further research is advised to confirm the effectiveness of topically applied ectoine and to define the most appropriate therapeutic protocols (i.e., treatment duration and dosage). Efficacy in the pediatric population has already been well described in the presented studies. No differences were found between $5.5 \%$ and $7 \%$ ectoine-containing formulations and creams or emollients; however, no directly comparative data were identified between ectoine concentration and formulation. Kudryavtseva and Migaliev included patients with cheilitis in their study and reported a high efficacy and safety profile for $7 \%$ ectoine containing cream [50]. Since cheilitis affects $80-90 \%$ of patients undergoing isotretinoin therapy [54], it would be interesting to include this indication in further research.

\section{CONCLUSIONS}

This systematic review of six studies extends our knowledge of ectoine and its potential supportive application in corticosteroid therapy by increasing efficacy in patients with atopic dermatitis. The majority of studies used SCORAD, a validated clinical assessment tool for atopic dermatitis, and assessed very sensitive patient groups, i.e., infants and children. Moreover, the combination of different treatment approaches, such as ectoine treatment in combination with other pharmacological therapy, showed additional potential for reducing the need for pharmacological therapy. The conclusion on ectoine efficacy on inflammatory skin diseases is also supported by evidence gained from other indications such as upper respiratory inflammation [43], allergic rhinitis [42], and irritation and inflammation of the eye surface [44]. No evidence on the efficacy of ectoine in other inflammatory skin diseases such as psoriasis could be identified, but this might be an interesting indication for further research. In addition, high-quality evidence, in the form of randomized controlled trials, might be useful.

\section{ACKNOWLEDGEMENTS}

Funding. Funding of the journal's Rapid Service Fee for this review was provided by bitop AG (Dortmund, Germany).

Medical Writing and Editorial Assistance. All authors contributed to the interpretation of data and conception of the manuscript 
and critically revised and approved the manuscript content. The authors thank Dr. Mira Woitok of IASON consulting GmbH \& Co. KG. (Niederzier, Germany) for providing medical writing and editorial support.

Authorship. All named authors meet the International Committee of Medical Journal Editors (ICMJE) criteria for authorship for this article, take responsibility for the integrity of the work as a whole, and have given their approval for this version to be published.

Author Contributions. Marion Kauth contributed by providing the concept and design as well as the drafting of the manuscript. Olga V. Trusova contributed by reviewing the medical background and proof-reading of the manuscript.

Disclosures. Marion Kauth is an employee of bitop AG. Olga V. Trusova declares that she has nothing to disclose.

Compliance with Ethics Guidelines. This article is based on previously conducted studies and does not contain any new studies with human participants or animals performed by any of the authors.

Data Availability. All data generated or analyzed during this study are included in this published article.

Open Access. This article is licensed under a Creative Commons Attribution-NonCommercial 4.0 International License, which permits any non-commercial use, sharing, adaptation, distribution and reproduction in any medium or format, as long as you give appropriate credit to the original author(s) and the source, provide a link to the Creative Commons licence, and indicate if changes were made. The images or other third party material in this article are included in the article's Creative Commons licence, unless indicated otherwise in a credit line to the material. If material is not included in the article's Creative Commons licence and your intended use is not permitted by statutory regulation or exceeds the permitted use, you will need to obtain permission directly from the copyright holder. To view a copy of this licence, visit http://creativecommons.org/licenses/by$\mathrm{nc} / 4.0 /$.

\section{REFERENCES}

1. Kubo A, Nagao K, Amagai M. Epidermal barrier dysfunction and cutaneous sensitization in atopic diseases. J Clin Investig. 2012;122(2):440-7.

2. Avena-Woods C. Overview of atopic dermatitis. Am J Manag Care. 2017;23:S115-23.

3. Silverberg JI, Hanifin JM. Adult eczema prevalence and associations with asthma and other health and demographic factors: a US population-based study. J Allergy Clin Immunol. 2013;132(5):1132-8.

4. Wollenberg A, et al. Consensus-based European guidelines for treatment of atopic eczema (atopic dermatitis) in adults and children: part I. J Eur Acad Dermatol Venereol. 2018;32(5):657-82.

5. Nutten S. Atopic dermatitis: global epidemiology and risk factors. Ann Nutr Metab. 2015;66(Suppl 1): 8-16.

6. Eichenfield LF, et al. Guidelines of care for the management of atopic dermatitis: section 1. Diagnosis and assessment of atopic dermatitis. J Am Acad Dermatol. 2014;70(2):338-51.

7. Drucker AM, et al. The burden of atopic dermatitis: summary of a report for the National Eczema Association. J Investig Dermatol. 2017;137(1): 26-30.

8. Silverberg NB, Silverberg JI. Inside out or outside in: does atopic dermatitis disrupt barrier function or does disruption of barrier function trigger atopic dermatitis? Cutis. 2015;96(6):359-61.

9. Wollenberg $\mathrm{A}$, et al. ETFAD/EADV Eczema task force 2020 position paper on diagnosis and treatment of atopic dermatitis in adults and children. J Eur Acad Dermatol Venereol. 2020;34(12): 2717-44.

10. Ridd MJ, et al. Best emollients for eczema (BEE) comparing four types of emollients in children with eczema: protocol for randomised trial and nested qualitative study. BMJ Open. 2019;9(11):e033387.

11. Kim MY, et al. Retinoid induces the degradation of corneodesmosomes and downregulation of corneodesmosomal cadherins: Implications on the 
mechanism of retinoid-induced desquamation. Ann Dermatol. 2011;23(4):439-47.

12. Ishida-Yamamoto A, Igawa S. The biology and regulation of corneodesmosomes. Cell Tissue Res. 2015;360(3):477-82.

13. van Zuuren EJ, et al. Emollients and moisturisers for eczema. Cochrane Database Syst Rev. 2017;2: CD012119.

14. Galinski EA, Pfeiffer HP, Truper HG. 1,4,5,6Tetrahydro-2-methyl-4-pyrimidinecarboxylic acid. A novel cyclic amino acid from halophilic phototrophic bacteria of the genus Ectothiorhodospira. Eur J Biochem. 1985;149(1):135-9.

15. Lentzen G, Schwarz T. Extremolytes: natural compounds from extremophiles for versatile applications. Appl Microbiol Biotechnol. 2006;72(4): 623-34.

16. Graf $\mathrm{R}$, et al. The multifunctional role of ectoine as a natural cell protectant. Clin Dermatol. 2008;26(4):326-633.

17. Harishchandra RK, et al. The effect of compatible solute ectoines on the structural organization of lipid monolayer and bilayer membranes. Biophys Chem. 2010;150(1-3):37-46.

18. Sydlik U, et al. The compatible solute ectoine protects against nanoparticle-induced neutrophilic lung inflammation. Am J Respir Crit Care Med. 2009;180(1):29-35.

19. Autengruber A, et al. Signalling-dependent adverse health effects of carbon nanoparticles are prevented by the compatible solute mannosylglycerate (firoin) in vitro and in vivo. PLoS One. 2014;9(11):e111485.

20. Abdel-Aziz $\mathrm{H}$, et al. Bacteria-derived compatible solutes Ectoine and $5 \alpha$-Hydroxyectoine act as intestinal barrier stabilizers to ameliorate experimental inflammatory bowel disease. J Nat Prod. 2015;78(6):1309-15.

21. Sydlik U, et al. Recovery of neutrophil apoptosis by ectoine: A new strategy against lung inflammation. Eur Respir J. 2013;41(2):433-42.

22. Peuschel H, et al. c-Src-mediated activation of Erk1/ 2 is a reaction of epithelial cells to carbon nanoparticle treatment and may be a target for a molecular preventive strategy. Biol Chem. 2010;391(11):1327-32.

23. Abdel-Aziz H, et al. Novel effects of ectoine, a bacteria-derived natural tetrahydropyrimidine, in experimental colitis. Phytomedicine. 2013;20(7): 585-91.
24. Bünger J, Degwert J, Driller $H$. The protective function of compatible solute ectoin on the skin cells and its biomolecules with respect to UV-radiation, immunosupression and membrane damage. IFSCC Mag. 2001;4(2):127-31.

25. Dwivedi $M$, et al. Biophysical investigations of the structure and function of the tear fluid lipid layer and the effect of ectoine. Part A: Natural meibomian lipid films. Biochim Biophys Acta. 2014;1838(10):2708-15.

26. Dwivedi $\mathrm{M}$, et al. Biophysical investigations of the structure and function of the tear fluid lipid layers and the effect of ectoine. Part B: Artificial lipid films. Biochim Biophys Acta. 2014;1838(10): 2716-27.

27. Harishchandra RK, et al. Compatible solutes: Ectoine and hydroxyectoine improve functional nanostructures in artificial lung surfactants. Biochim Biophys Acta. 2011;1808(12):2830-40.

28. Harishchandra RK, Saleem M, Galla H-J. Nanoparticle interaction with model lung surfactant monolayers. J R Soc Interface. 2010;7 Suppl 1: S15-26.

29. Kroker M, et al. Preventing carbon nanoparticleinduced lung inflammation reduces antigen-specific sensitization and subsequent allergic reactions in a mouse model. Part Fibre Toxicol. 2015;12:20.

30. Smiatek J, et al. Properties of compatible solutes in aqueous solution. Biophys Chem. 2012;160(1): $62-8$.

31. Unfried $\mathrm{K}$, et al. The compatible solute ectoine reduces the exacerbating effect of environmental model particles on the immune response of the airways. J Allergy. 2014;2014:708458.

32. Sonnemann U, Scherner O, Werkhäuser N. Treatment of rhinitis sicca anterior with ectoine containing nasal spray. J Allergy. 2014;2014:273219.

33. Werkhäuser N, Bilstein A, Sonnemann U. Treatment of allergic rhinitis with ectoine containing nasal spray and eye drops in comparison with azelastine containing nasal spray and eye drops or with cromoglycic acid containing nasal spray. J Allergy. 2014;2014:176597.

34. Eichel A, et al. A prospective, controlled study of SNS01 (ectoine nasal spray) compared to BNO-101 (phytotherapeutic dragées) in patients with acute rhinosinusitis. Curr Med Res Opin. 2013;29(7): 739-46.

35. Marini A, et al. Ectoine-containing cream in the treatment of mild to moderate atopic dermatitis: a randomised, comparator-controlled, intra- 
individual double-blind, multi-center trial. Skin Pharmacol Physiol. 2014;27(2):57-65.

36. Unfried $\mathrm{K}$, et al. Reduction of neutrophilic lung inflammation by inhalation of the compatible solute ectoine: a randomized trial with elderly individuals. Int J Chronic Obstr Pulm Dis. 2016;11: 2573-83.

37. Dao VA, et al. Effectiveness, tolerability, and safety of Ectoine-containing mouthwash versus those of a calcium phosphate mouthwash for the treatment of chemotherapy-induced oral mucositis: a prospective, active-controlled, non-interventional study. Oncol Ther. 2018;6(1):59-72.

38. Dao VA, et al. Ectoine lozenges in the treatment of acute viral pharyngitis: a prospective, active-controlled clinical study. Eur Arch Otorhinolaryngol. 2019;276(3):775-83.

39. Muller D, et al. Efficacy and tolerability of an ectoine mouth and throat spray compared with those of saline lozenges in the treatment of acute pharyngitis and/or laryngitis: a prospective, controlled, observational clinical trial. Eur Arch Otorhinolaryngol. 2016;273(9):2591-7.

40. Salapatek AM, et al. Effects of ectoine containing nasal spray and eye drops on symptoms of seasonal allergic rhinoconjunctivitis. Clin Transl Allergy. 2021;11(1):e12006.

41. Tran $\mathrm{BH}$, et al. Ectoine-containing inhalation solution versus saline inhalation solution in the treatment of acute bronchitis and acute respiratory infections: a prospective, controlled, observational study. Biomed Res Int. 2019;2019:7945091.

42. Eichel A, et al. Meta-analysis of the efficacy of ectoine nasal spray in patients with allergic rhinoconjunctivitis. J Allergy. 2014;2014:292545.

43. Casale $\mathrm{M}$, et al. Topical ectoine: a promising molecule in the upper airways inflammation-a systematic review. Biomed Res Int. 2019;2019:7150942.

44. Bilstein A, et al. Ectoine in the treatment of irritations and inflammations of the eye surface. Biomed Res Int. 2021;2021:8885032.
45. Page MJ, et al. The PRISMA 2020 statement: an updated guideline for reporting systematic reviews. PLoS Med. 2021;18(3):e1003583.

46. Page MJ, et al. PRISMA 2020 explanation and elaboration: updated guidance and exemplars for reporting systematic reviews. BMJ. 2021;372:n160.

47. Schardt C, et al. Utilization of the PICO framework to improve searching PubMed for clinical questions. BMC Med Inform Decis Mak. 2007;7:16.

48. Wilkowska A, Grubska-Suchanek E, Nowicki R. Evaluation of safety and efficacy of Dermaveel in treatment of atopic dermatitis. Alergol Pol Pol J Allergol. 2015;2(4):128-33.

49. Hon KL, et al. Testing an ectoin containing emollient for atopic dermatitis. Curr Pediatr Rev. 2019;15(3):191-5.

50. Kudryavtseva A, Mingaliev R. Dry skin as an auxiliary diagnostic criterion for atopic dermatitis. Experience in effectively using ectoin-containing external agent in childhood. Vrach. 2019;30(3): $30-4$.

51. Trusova OV, et al. Prospective open controlled study of $7 \%$ ectoine cream in complex treatment of persistent skin lesions in children with atopic dermatitis. Klin Dermatol Venerol. 2019;18(5):583-90.

52. Tlish MM, Shavilova ME. Modern approaches to the prevention and correction of complications caused by systemic treatment of severe acne forms. Vestn Sermatol Venerol. 2019;95(2):87-93.

53. Diaz-Sanchez D, Penichet-Garcia M, Saxon A. Diesel exhaust particles directly induce activated mast cells to degranulate and increase histamine levels and symptom severity. J Allergy Clin Immunol. 2000;106(6):1140-6.

54. Romiti R, Romiti N. Dexpanthenol cream significantly improves mucocutaneous side effects associated with isotretinoin therapy. Pediatr Dermatol. 2002;19(4):368. 